and the age estimates based on comparative abundances of radioactive nuclei. This would at least avoid the problems created by the higher value of $H_{0}$ of $100 \mathrm{~km} \mathrm{~s}^{-1} \mathrm{Mpc}^{-1}$, which suggests a Universe age of nine billion years com- pared with star cluster ages which are estimated to be in excess of ten billion years. The lower value of $50 \mathrm{~km} \mathrm{~s}^{-1} \mathrm{Mpc}^{-1}$ cannot be ruled out, although the age of galaxies (following Osmer's result) would have to be at least 14.5 billion years.

\section{0th anniversary of Gauss's first absolute magnetic measurement}

\author{
from S.R.C. Malin and D.R. Barraclough
}

ON 26 May 1832, the horizontal component of the Earth's magnetic field at Göttingen was $1.7820 \mathrm{mg}^{1 / 2} \mathrm{~mm}^{-1 / 2} \mathrm{~s}^{-1}$ $(=17,820 \mathrm{nT})$. Not particularly remarkable, you might think, except that this was the first ever absolute measurement of the geomagnetic field and its attainment had required much of the armoury of one of the world's greatest mathematicians.

Carl Friedrich Gauss (1777-1855) was a gifted child. His early mathematical feats led his father grudgingly to agree with his teachers that the boy should receive higher education rather than learn a trade, but Gauss was also an exceptional linguist and might equally easily have chosen to study philology. It was the satisfaction he derived in 1796 from demonstrating the construction of a regular 17-sided polygon with ruler and compasses that finally decided him on a career in mathemtics. In his doctoral thesis, he proved the 'fundamental theorem of algebra' (the first of his four independent proofs of this theorem), and most of his work at this time was similarly concerned with pure mathematics. Although this continued to be his main interest, he gradually branched out into many other subjects, including applied mathematics, astronomy, geodesy, optics, telegraphy and geomagnetism.

Soon after 1830 , Gauss became interested in the adoption of a universal system of units for all physical quantities, and came to the remarkable conclusion that even magnetic intensity could be measured in terms of mass, length and time. With the practical assistance of Wilhelm Weber, professor of physics in the University of Göttingen, he set about devising an experiment to make this measurement. He explains some of his ideas in a letter to the astronomer Olbers written on 18 February 1832.

"I occupy myself now with the Earth's magnetism, particularly with an absolute determination of its intensity. Friend Weber conducts the experiments on my instructions. As, for example, a clear concept of velocity can be given only through statements on time and space, so in my opinion, the complete determination of the intensity of the Earth's magnetism requires to specify (1) a weight $=p$, (2) a length $=r$, and then the Earth's magnetism can be expressed by $\sqrt{ } p / r$."

The experiment came to fruition three months later, after Gauss had slightly revised his dimensional arguments. The apparatus required is very simple - two bar magnets (A and B), one single-filament suspension in which either magnet can be fitted, a ruler, a clock and two weights. The method is also simple, though a number of refinements are required if its full accuracy is to be realized. The first part is the vibration experiment in which magnet $A$ is suspended horizontally, and its period of oscillation either side of magnetic north measured. The period $T$ is related to the magnet's moment of inertia, $I$, its magnetic moment, $M$, and the horizontal intensity of the geomagnetic field, $H$, by

$$
T=2 \pi \sqrt{\frac{I}{M H}}
$$

Thus, if $I$ is known, we can infer $M H$. Gauss deduced $I$ by adding known increments to it in the form of weights at known distances from the suspension, and noting how this affected $T$. In calculating $I$ from the several different values of $T$ and $\Delta I$, Gauss used a powerful technique which he had developed himself, the method of least squares. He also took care to adjust $T$ to the value it would attain with vibrations of infinitesimal amplitude, as the equation is strictly valid only for that case.

The second part is the deflection experiment in which the ratio $M / H$ is determined by suspending magnet $\mathrm{B}$ and noting its angular deflection from magnetic north when magnet $A$ is placed at a known distance to the east or west. This requires a knowledge of the way in which the magnetic intensity due to a bar magnet falls off with distance. Gauss deduced this from first principles, showing not only that the intensity due to a dipole depends on the inverse cube of the distance, but also that one additional term is both necessary and sufficient to allow for the finite length of the bar magnet. He determined the parameters by measuring the deflection with magnet $A$ at different distances, again solving by the method of least squares.

From a knowledge of $M H$ and $M / H$,

S.R.C. Malin and D.R. Barraclough are in the Institute of Geological Sciences, Murchison House, West Mains Road, Edinburgh EH9 $3 L A$. both $M$ and $H$ can readily be deduced.

Because of secular change, it is difficult to assess the accuracy of Gauss's original observation but, from internal consistency and backward extrapolation of the trend from later observations, it seems likely that it was within one per cent of the true value. With only minor modifications, Gauss's method continued to be the standard way of measuring $\mathrm{H}$ at magnetic observatories and in the field until the $1920 \mathrm{~s}$, and it is still in use at some observatories. Nowadays, absolute magnetic intensity measurements are usually made with a proton magnetometer. This gives the answer to an accuracy of about 1 part in $10^{6}$ in a few seconds.

This was not Gauss's only contribution to geomagnetism. He later applied yet another of his inventions, the method of spherical harmonic analysis, to the Earth's magnetic field to show that nearly all of it came from inside the Earth. He was also, with Humboldt, largely responsible for the 'Gottingen Magnetic Union', an international project for the simultaneous observation of the magnetic field on four selected term-days each year at a number of widespread sites. This scheme developed into the worldwide network of magnetic observatories that exists today.

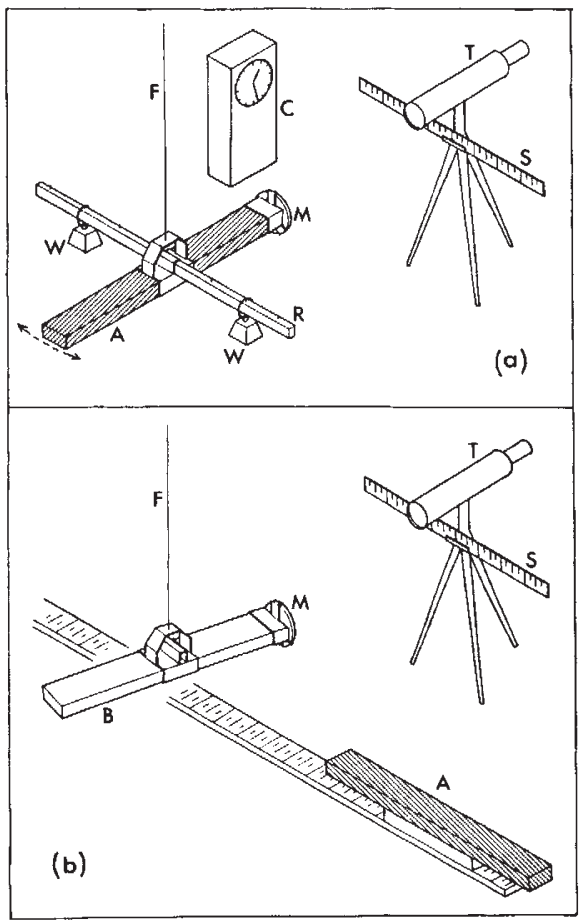

Gauss's experiment. (a) The vibration experiment. The magnet, A, suspended by a silk thread, $\mathbf{F}$, oscillates in a horizontal plane. Its moment of inertia can be varied by hanging weights, W, from the rod, $\mathbf{R}$. The period of oscillation is obtained by observing the reflection of the scale, $\mathrm{S}$, in mirror $\mathrm{M}$, through the telescope, $\mathrm{T}$, and timing an integral number of swings with the clock, C. (b) The deflection experiment. Magnet B is suspended and is deflected from magnetic north by placing magnet $\mathrm{A}$ at a known distance. The deflection is measured by observing the reflection of the scale, $\mathrm{S}$, in mirror $\mathrm{M}$, through the telescope, $\mathrm{T}$. 